\title{
Assessment of Near-Earth Asteroid Deflection Techniques via Spherical Fuzzy Sets
}

\author{
M. Fernández-Martínez (iD) and J. M. Sánchez-Lozano \\ University Centre of Defence at the Spanish Air Force Academy, MDE-UPCT, 30720-Santiago De La Ribera, \\ Región De Murcia, Spain \\ Correspondence should be addressed to M. Fernández-Martínez; manuel.fernandez-martinez@cud.upct.es
}

Received 23 October 2020; Revised 22 January 2021; Accepted 18 February 2021; Published 8 March 2021

Academic Editor: Maria Gritsevich

Copyright @ 2021 M. Fernández-Martínez and J. M. Sánchez-Lozano. This is an open access article distributed under the Creative Commons Attribution License, which permits unrestricted use, distribution, and reproduction in any medium, provided the original work is properly cited.

\begin{abstract}
Extensions of fuzzy sets to broader contexts constitute one of the leading areas of research in the context of problems in artificial intelligence. Their aim is to address decision-making problems in the real world whenever obtaining accurate and sufficient data is not a straightforward task. In this way, spherical fuzzy sets were recently introduced as a step beyond to modelize such problems more precisely on the basis of the human nature, thus expanding the space of membership levels, which are defined under imprecise circumstances. The main goal in this study is to apply the spherical fuzzy set version of Technique for Order of Preference by Similarity to Ideal Solution (TOPSIS), a well-established multicriteria decision-making approach, in the context of planetary defense. As of the extraction of knowledge from a group of experts in the field of near-Earth asteroids, they rated four deflection technologies of asteroids (kinetic impactor, ion beam deflection, enhanced gravity tractor, and laser ablation) that had been previously assessed by means of the classical theory of fuzzy series. This way, a comparative study was carried out whose most significant results are the kinetic impactor being the most suitable alternative and the spherical fuzzy set version of the TOPSIS approach behaves more sensitively than the TOPSIS procedure for triangular fuzzy sets with regard to the information provided by our group of experts.
\end{abstract}

\section{Introduction}

Meteoroids reach the Earth mainly as small rocks and fragile aggregates which appear as a consequence of the decay of asteroids and comets. In this way, the tiny dusts that arrive at the Earth each day amounts to a mean of approximately 100 tons [1].

Although it is true that larger objects will unlikely reach Earth's orbit, a potential impactor may dramatically affect the life and the climate in our planet. For instance, a $10 \mathrm{~km}$ wide impactor led to the so-called Cretaceous-Paleocene extinction that happened 64 million years ago [2, 3]. In 1908, the fragmentation at a low altitude $(5-10 \mathrm{~km})$ of an asteroid with an estimated diameter $d \in[30,50] \mathrm{m}$ destroyed around $2000 \mathrm{~km}^{2}$ of woodland [4], what was known as the Tunguska event. Recently, in 2013, a bolide with an estimated weight of 12000 tons and an estimated diameter of $19 \mathrm{~m}$ entered Earth's atmosphere at a relative velocity of $19 \mathrm{~km} / \mathrm{s}$. The object broke up at an altitude of $30 \mathrm{~km}$, thus injuring more than 1500 people $[2,5,6]$.

The latter are clear examples of fatal consequences that impact of these kinds of objects with the Earth may result in. The impact of a single massive cosmic body might leave a fairly large crater in the surface of the Earth or induce a tsunami in the case it collides with the surface of the ocean, thus contributing to overall risk [7-10]. Furthermore, air blasts derived from destruction of meteoritic bodies in Earth's atmosphere may provoke not only falls of clouds of fragments but also major injuries on Earth's surface even if the object does not make a touchdown. Indeed, such explosions may cause from glass breakage (for air blasts greater than $15 \mathrm{~m} / \mathrm{s}$ ) to extreme distortions in steel structures of bridges or buildings which may lead to their collapse (for air blasts greater than $200 \mathrm{~m} / \mathrm{s}$ ) [11] and ([12], Table 1). Therefore, such consequences do not only depend on the size of the impactor but also on other parameters such as entry angle, velocity, density, and shape [12]. 
Near-Earth objects (NEOs) are asteroids or comets whose perihelion distance is less than 1.3 AU (about 195 million $\mathrm{km}$ ). Short period comets with orbital periods less than 200 years are known as near-Earth comets (NECs) whose orbits lie far away from the Earth. In this way, researchers are mainly focused on the tracking of the nearEarth asteroids (NEAs) [2, 13].

Three key reasons that support the study of NEAs are, namely, planetary defense, scientific knowledge (e.g., deepen our solar system origins), and mining. In this regard, the current study is allocated to planetary defense.

In particular, the so-called potentially hazardous asteroids are NEAs whose minimum orbit interception distance with respect to Earth's orbit is less than $0.05 \mathrm{AU}$. They are also characterized by an estimated size greater than $140 \mathrm{~m}$ in diameter and an absolute magnitude not greater than 22.0 , as well. Since they are able to closely approach Earth's orbit, small perturbations regarding their orbits may place them on a collision path to the Earth. As such, to perform an exhaustive tracking of these asteroids is recommended $[14,15]$.

Some efforts have already been made with the aim of redirecting a NEA out of a risky trajectory with the Earth', at least at a first glance. Indeed, in 2013, the NASA introduced the Asteroid Redirect Mission (ARM) with a double purpose, namely, redirect small (i.e., less than $8 \mathrm{~m}$ diameter) asteroids and extract small (less than $4 \mathrm{~m}$ ) boulders from the surface of a wide asteroid and place it on a distant retrograde orbit around the moon [16]. The later could be understood as a preliminary test of the current technical capacity to deliver and catch an object to a safe environment, despite that mission did not actually consist of redirecting a small PHA. However, that technological and scientific project was cancelled in 2017 [17].

Notwithstanding, several encouraging approaches for NEA redirection have been posed, even though the technology underlying them has not yet completely developed. In this regard, we would like to highlight the Double Asteroid Redirection Test (DART) that will be the first in situ exhibition of a kinetic impactor to deflect an asteroid in space. This was formerly applied to the satellite of the binary NEA (65803) Didymos [18].

Redirecting an asteroid should be distinguished from deflecting it. In fact, deflecting an asteroid consists of modifying its trajectory to avert a potential impact with the Earth. As such, nuclear blast and kinetic impactor are examples of deflection techniques. On the other hand, the aim of redirecting an object is to induce a controlled change in its orbit with a further purpose, as it is the case of laser ablation/ sublimation, tugboat, mass driver, and ion beam ([2] and references therein). However, in this study, we shall understand a deflection technology as that one being able of deflecting or redirecting an asteroid.

It is worth pointing out that, in [19], an assessment involving four deflection technologies for asteroids smaller than 250 meters in diameter (a range of sizes that covers most of the impactors with the Earth that occur in timescales up to 100000 years), i.e., kinetic impactor, ion beam deflection, enhanced gravity tractor, and laser ablation, was carried out in regard to eight criteria (build time, level of maturity of a NEA deflection technology, asteroid rotation, asteroid structure, asteroid composition, asteroid shape, active deflection duration, and mission risk). Such a decision problem was addressed throughout a combination of fuzzy logic and multicriteria decision-making (MCDM, hereafter) approaches.

We recall that a MCDM problem consists of looking for the best choice from a set of alternatives by a set of criteria, and to deal with, all that information is arranged into the socalled decision matrix [20]. A wide collection of MCDM algorithms, such as ELECTRE, OWA, VIKOR, ANP, and, PROMETHEE, can be found in the literature.

Despite MCDM methodologies had been previously applied to address several issues regarding NEAs ([20, 21]), they were first combined with fuzzy logic recently in [19], where four NEA deflection technologies (described in Section 3.2) were rated with respect to a set consisting of eight criteria (Section 3.3). The involvement of fuzzy logic therein was mainly motivated by the existence of qualitative criteria whose values are difficult to be specified or measured. In this way, linguistic labels that were associated with triangular fuzzy numbers, as well as (the valuable knowledge from) the judgments provided by an international board consisting of great standing scientists in the field of NEAs, were used to quantify the level of importance of such criteria. This way, the level of importance of each criterion was calculated, thus leading to solve the decision problem that had been posed by means of the MCDM approach named Technique for Order of Preference by Similarity to Ideal Solution (TOPSIS).

Zadeh was the first to introduce that approach to manage uncertainty and ambiguity when there exist attributes that are hard to be quantified [22]. In fuzzy logic, the level of membership of an element to a series is determined by a real number lying in the interval $[0,1]$, thus leading to a fuzzy series. Since that pioneer work, fuzzy series have been applied not only to deal with decision problems in a wide range of contexts (e.g., [23]) but also to contribute new viewpoints in that field including intuitionistic fuzzy sets [24], Pythagorean fuzzy sets [25-28], neutrosophic fuzzy sets [29], or picture fuzzy sets $[30,31]$.

Extensions of fuzzy sets to broader contexts constitute one of the leading areas of research in the context of problems in computational intelligence. Their aim is to address decision-making problems in the real world whenever obtaining accurate and sufficient data is not a straightforward task. In this way, spherical fuzzy sets were recently introduced as a step beyond the picture fuzzy sets to modelize MCDM problems more precisely on the basis of the human nature, thus expanding the space of membership levels, which are defined under imprecise circumstances [32]. Even though they were introduced recently [33], it is worth mentioning that several operators, distance measures, and even some applications have already been contributed [34-38].

Being encouraged by such novel contributions, we wondered to what extent ranking of alternatives would vary if either fuzzy sets are considered to deal with a MCDM 
problem or the most recent extensions of fuzzy sets (such as spherical fuzzy sets) are used with the same purpose. Following the above, this study addresses that question by applying the spherical fuzzy version of the TOPSIS approach to a decision problem that had previously been posed in the context of planetary defense [19].

The structure of this study is as follows. Section 2 contains the basics on fuzzy sets and fuzzy logic (Section 2.1), spherical fuzzy sets and their operators (Section 2.2), and also includes a description regarding the generalization of the TOPSIS approach to the context of spherical fuzzy sets (Section 2.3). The assumptions of our study are summarized in Section 3.1. Next, we recall the four NEA deflection technologies to be evaluated in this study (Section 3.2) together with the selected criteria (Section 3.3). Furthermore, some comments on the board of experts who provided us valuable information to determine the weights of the criteria are provided in Section 3.4. Our results and discussion are provided in Section 4, whereas two analyses of sensitivity are carried out and further discussed in Section 5. Finally, the main conclusions of this study are presented in Section 6 .

\section{Methodology}

2.1. On Fuzzy Sets and Fuzzy Logic. Fuzzy logic constitutes an alternative to classical logic to deal with decision making by introducing some degree of vagueness to assess situations or objects.

In 1965, membership functions and fuzzy sets were mathematically introduced to model the level of incertitude and ambiguity in regard to human thinking [22]. In this way, the domain of a membership function turns into the unit interval $[0,1]$ rather than the set $\{0,1\}$. As such, in the context of the classical logic, the membership of an element to a set is completely determined, whereas in the fuzzy logic, such a membership could be measured gradually.

The application of fuzzy logic to real-life contexts results especially appropriate when the rules of membership of a given element to a certain class cannot be stated clearly [39]. In fact, the category itself may depend on the context.

In fuzzy logic, the level of membership of an element to a class is quantified by a real number that belongs to the interval $[0,1]$. In this way, if the membership level of an element to a certain set is close to 1 , then it is more likely that such an element belongs to that class. On the contrary, if that degree of membership is close to 0 , then it is more unlikely that it belongs to that set.

Let $A \subseteq U$, where $U$ refers to a universe of discourse. A membership function can be defined as a rule of association, $\mu_{A}: U \longrightarrow[0,1]$, that maps every $x \in U$ to its degree of membership to $A, \mu_{A}(x) \in[0,1]$. Hence, the concept of a membership function can be further extended to a qualitative setting by means of linguistic labels and variables that are more accurate than crisp numbers in such contexts [40]. Reciprocally, each function $\mu: U \longrightarrow[0,1]$ allows defining a membership function that is associated to a certain fuzzy set, thus depending on the context it is applied to and the concept it represents. In this way, several functions have been widely applied including the Gaussian, the PI (or trapezoidal), and the LAMBDA (or triangular) ones.

Several extensions of ordinary fuzzy sets have appeared in the literature (e.g., [33] for a chronological tracking of them). Among them, we would like to highlight those generalizations of fuzzy sets with a three-dimensional membership function.

An intuitionistic fuzzy set is one of the form $\widetilde{A}=\left\{\left\langle\mu_{\widetilde{A}}(u), \nu_{\tilde{A}}(u)\right\rangle: u \in U\right\}$, where $\mu_{\widetilde{A}}: U \longrightarrow[0,1]$ is the membership function that quantifies the degree of membership of each element $u$ to $\widetilde{A}$, and $\nu_{\widetilde{A}}: U \longrightarrow[0,1]$ is the nonmembership function. They satisfy that $\mu_{\tilde{A}}(u)+$ $\nu_{\mathcal{A}}(u) \in[0,1]$ for all $u \in U$. In addition, $\pi_{\mathcal{A}}^{\sim}(u)=$ $1-\mu \widetilde{A}(u)-\nu_{\mathcal{A}}(u)$ is defined as the degree of hesitancy of $u$ to $\widetilde{A}$. However, in real-life applications, it may happen that, for a certain alternative satisfying a criterion, the sum of the squares of the membership and nonmembership functions stands not greater than 1 with their sum being greater than 1 . With the aim to avoid the experts modifying their preferences, the second type intuitionistic fuzzy sets were introduced by Atanassov in [41]. They are the form $\widetilde{A}=\left\{\left(\mu_{\widetilde{A}}(u), \nu_{\widetilde{A}}(u)\right): u \in U\right\}$, where its membership function, $\mu_{A}: U \longrightarrow[0,1]$, and its nonmembership function, $\nu_{\widetilde{A}}: U \stackrel{A}{\longrightarrow}[0,1]$, satisfy that $\mu_{\widetilde{A}}^{2}(u)+v_{\widetilde{A}}^{2}(u) \in[0,1]$ for all $u \in U$. In addition, the degree ${ }^{A}$ of hesitancy of each $u \in U$ with respect to $\widetilde{A}$ is given by the following expression:

$$
\pi_{A}^{\sim}(u)=\left(1-\mu_{\tilde{A}}^{2}(u)-v_{\tilde{A}}^{2}(u)\right)^{1 / 2} .
$$

We would like also to point out that further generalizations of the TOPSIS approach under fuzziness have been proposed in the literature on the context of interval-valued spherical fuzzy sets ([42]).

2.2. Spherical Fuzzy Sets and Operators. Going beyond, in ([33], Definition 3), the spherical fuzzy sets were first introduced to allow the hesitancy of a decision maker be defined independently of her/his degrees of membership and nonmembership in regard to an alternative with respect to a criterion. Their definition, which appears in this section, consists of using the Euclidean distance on a spherical volume rather than measuring arc distances on the surface of a sphere, as it was proposed in $[43,44]$.

Next, we recall how to define them. Let $U$ be a universe of discourse. A spherical fuzzy set (SFS, hereafter) of $U$ is a set of the form

$$
\widetilde{A}_{S}=\left\{\left\langle\mu{\tilde{A_{S}}}(u), \nu_{\tilde{A}_{S}}(u), \pi{\widetilde{A_{S}}}(u)\right\rangle: \quad u \in U\right\},
$$

where $\mu_{\tilde{A}_{S}}, \nu_{\widetilde{A}_{S}}$, and $\pi{\widetilde{A_{S}}}_{\mathrm{s}}: U \longrightarrow[0,1]$ are the functions that quantify the degree of membership, nonmembership, and hesitancy of each $u \in U$ to the SFS $\widetilde{A}_{S}$, respectively. They satisfy that $\mu_{\widetilde{A}_{s}}^{2}(u)+v_{\widetilde{A}_{S}}^{2}(u)+\pi_{\widetilde{A}_{s}}^{2}(u) \in[0,1]$ for all $u \in U$.

Let $\varepsilon=\left\langle\mu \widetilde{A}_{A_{S}}^{A_{S}}(u), v \sim_{A_{S}}^{A_{S}}(u), \pi_{\tilde{A}_{S}}{ }^{A_{S}}(u)\right\rangle: u \in U$ be a spherical fuzzy number (SFN, hereafter). The product of $\varepsilon$ by a scalar $\lambda>0$ was defined as follows: 


$$
\lambda \cdot \varepsilon=\left\{\left\langle\left(1-\left(1-\mu_{\tilde{A}_{S}}^{2}(u)\right)^{\lambda}\right)^{1 / 2}, \nu{\widetilde{A_{S}}}_{(}^{\lambda}(u),\left(\left(1-\mu_{\tilde{A}_{S}}^{2}(u)\right)^{\lambda}-\left(1-\mu_{\tilde{A}_{S}}^{2}(u)-\pi_{\tilde{A}_{S}}^{2}(u)\right)^{\lambda}\right)^{1 / 2}\right\rangle: \quad u \in U\right\},
$$

whereas the $\lambda$ - power of $\varepsilon$ is given by

$$
\varepsilon_{S}^{\lambda}=\left\{\left\langle\mu \tilde{A}_{\tilde{A}_{S}}^{\lambda}(u),\left(1-\left(1-v_{\tilde{A}_{S}}^{2}(u)\right)^{\lambda}\right)^{1 / 2},\left(\left(1-v_{\tilde{A}_{S}}^{2}(u)\right)^{\lambda}-\left(1-v_{\widetilde{A}_{S}}^{2}(u)-\pi_{\widetilde{A}_{S}}^{2}(u)\right)^{\lambda}\right)^{1 / 2}\right\rangle: \quad u \in U\right\} .
$$

([33], Definition 5). We also refer the reader to ([33], Definition 6) for some properties regarding products of SFS by scalars (with respect to $\oplus$ ) and powers of SFSs (with respect to the operator $\otimes$.)

On the other hand, let $\omega=\left(\omega_{1}, \omega_{2}, \ldots, \omega_{n}\right)$ be a normalized list of weights, i.e., $\omega_{i} \in[0,1]$ for all $i=1,2, \ldots, n$ with $\sum_{i=1}^{n} \omega_{i}=1$. It is worth mentioning that several operators for SFNs were introduced in ([33], Section 3). Next, we recall the definitions of spherical weighted arithmetic mean (SWAM, ([33], Definition 7)) and spherical weighted geometric mean (SWGM, [33], Definition 8)) operators with respect to a normalized list of weights that will be used in this study. Let $\left\{\varepsilon_{i}: i=1, \ldots, n\right\}$ be a finite list of triangular fuzzy numbers, where $\varepsilon_{i}=\left\langle\mu_{\widetilde{A}_{s_{i}}}(u), \nu_{\widetilde{A}_{s_{i}}}(u), \pi_{\widetilde{A}_{s_{i}}}(u)\right\rangle$ for each $i=1, \ldots, n$. Then,

$$
\begin{aligned}
& \operatorname{SWAM}_{\omega}\left(\varepsilon_{1}, \ldots, \varepsilon_{n}\right):=\sum_{i=1}^{n} \omega_{i} \varepsilon_{i}=\omega_{1} \varepsilon_{1}+\cdots+\omega_{n} \varepsilon_{n} \\
& =\left\{\left\langle\left[1-\prod_{i=1}^{n}\left(1-\mu_{\widetilde{A}_{S_{i}}}^{2}(u)\right)^{\omega_{i}}\right]^{1 / 2}, \prod_{i=1}^{n} \nu_{\widetilde{A}_{S_{i}}}^{\omega_{i}}(u),\left[\prod_{i=1}^{n}\left(1-\mu_{\widetilde{A}_{S_{i}}}^{2}(u)\right)^{\omega_{i}}-\prod_{i=1}^{n}\left(1-\mu_{\widetilde{A}_{S_{i}}}^{2}(u)-\pi_{\widetilde{A}_{S_{i}}}^{2}(u)\right)^{\omega_{i}}\right]^{1 / 2}\right\rangle: u \in U\right\}, \\
& \operatorname{SWGM}_{\omega}\left(\varepsilon_{1}, \ldots, \varepsilon_{n}\right):=\sum_{i=1}^{n} \varepsilon_{i}^{\omega_{i}}=\varepsilon_{1}^{\omega_{1}}+\cdots+\varepsilon_{n}^{\omega_{n}} \\
& =\left\{\left\langle\prod_{i=1}^{n} \mu_{\tilde{A}_{S_{i}}}^{\omega_{i}}(u),\left[1-\prod_{i=1}^{n}\left(1-v_{\tilde{A}_{S_{i}}}^{2}(u)\right)^{\omega_{i}}\right]^{1 / 2},\left[\prod_{i=1}^{n}\left(1-v_{\tilde{A}_{S_{i}}}^{2}(u)\right)^{\omega_{i}}-\prod_{i=1}^{n}\left(1-v_{\tilde{A}_{S_{i}}}^{2}(u)-\pi_{\tilde{A}_{S_{i}}}^{2}(u)\right)^{\omega_{i}}\right]^{1 / 2}\right\rangle: u \in U\right\} .
\end{aligned}
$$

Finally, for a SFN, $\varepsilon=\left\langle\mu_{\tilde{A}_{s}}(u), \nu_{\tilde{A}_{c}}(u), \pi_{\widetilde{A}_{s}}(u)\right\rangle: u \in U$, recall that its score was defined in the following terms ([33], Definition 9):

$$
\operatorname{Score}(\varepsilon)=\left(\mu_{\widetilde{A}_{S}}(u)-\pi_{\widetilde{A}_{S}}(u)\right)^{2}-\left(v_{\widetilde{A}_{S}}(u)-\pi_{\widetilde{A}_{S}}(u)\right)^{2} .
$$

2.3. The SFS TOPSIS. Interestingly, some extensions of fuzzy sets have led to new versions of the TOPSIS approach (e.g., [33] for a literature review concerning them). In this study, we shall apply the SFS version of the TOPSIS approach (SFS TOPSIS, hereafter) that was first introduced ([33], Section 5) and already applied in the literature (e.g., $[45,46])$.

First, recall that a MCDM problem can be expressed by a decision matrix whose entries contain the evaluation of the alternatives with respect to each criterion. Thus, first, let $m \geq 2, X=\left\{X_{1}, X_{2}, \ldots, X_{m}\right\}$ be a finite set of alternatives, $C=\left\{C_{1}, C_{2}, \ldots, C_{n}\right\}$ be a discrete set of criteria, and $\omega=$ $\left(\omega_{1}, \omega_{2}, \ldots, \omega_{n}\right)$ be a normalized list of weights, i.e., $\omega_{i} \in[0,1]$ for all $i=1,2, \ldots, n$ and $\sum_{i=1}^{n} \omega_{i}=1$. Then, that decision matrix constitutes the starting point to apply the SFS TOPSIS approach, which includes the following stages.

Step 1: the evaluation matrices of alternatives and criteria have to be filled in by the decision makers. With this aim, the linguistic labels that appear in Table 1 should be used.

Step 2: the judgments of the decision makers have to be aggregated by means of the SWAM (respectively, the SWGM) operator as defined above. Specifically,

Step 2.1: the individual valuations of the decision makers in regard to the relative importance of each criterion have to be combined to obtain the weights of the criteria.

Step 2.2: construction of the aggregated spherical fuzzy decision matrix by taking into account the judgments of the decision makers. In fact, let us denote the evaluation of the alternative $X_{i}$ with respect to the criterion $C_{j}$ by $C_{j}\left(X_{i}\right)=\left(\mu_{i j}, v_{i j}, \pi_{i j}\right)$ for all $i=1, \ldots, m$ and all $j=1, \ldots, n$. Hence, let $D:=\left(C_{j}\left(X_{i}\right)\right)_{m \times n}$ be the decision matrix of a SFS MCDM problem. 
TABLE 1: Linguistic terms and their associated linguistic labels and SFNs $(\mu, \nu, \pi)$.

\begin{tabular}{|c|c|c|c|c|}
\hline Linguistic term & Label & $\mu$ & $v$ & $\pi$ \\
\hline Absolutely more importance & AMI & 0.9 & 0.1 & 0.1 \\
\hline Very high importance & VHI & 0.8 & 0.2 & 0.2 \\
\hline High importance & $\mathrm{HI}$ & 0.7 & 0.3 & 0.3 \\
\hline Slightly more importance & SMI & 0.6 & 0.4 & 0.4 \\
\hline Equally importance & EI & 0.5 & 0.5 & 0.5 \\
\hline Slightly low importance & SLI & 0.4 & 0.6 & 0.4 \\
\hline Low importance & LI & 0.3 & 0.7 & 0.3 \\
\hline Very low importance & VLI & 0.2 & 0.8 & 0.2 \\
\hline Absolutely low importance & ALI & 0.1 & 0.9 & 0.1 \\
\hline
\end{tabular}

Step 3: construction of the aggregated weighted spherical fuzzy decision matrix. Once the alternatives have been ranked and the weights of the criteria determined, calculate $\widetilde{D}=\left(C_{j}\left(X_{i \omega}\right)\right)_{m \times n}$, where

$C_{j}\left(X_{i \omega}\right)=\left(\mu_{i j \omega}, v_{i j \omega}, \pi_{i j \omega}\right)$ for all $i=1, \ldots, m$ and all $j=1, \ldots, n$. Notice that ([33], equation (14)) is applied in this step.

Step 4: defuzzification of the aggregated weighted spherical fuzzy decision matrix is by applying the score function (equation (6)). To tackle with, use Score $\left(C_{j}\left(X_{i \omega}\right)\right)=\left(\mu_{i j \omega}-\pi_{i j \omega}\right)^{2}-\left(\nu_{i j \omega}-\pi_{i j \omega}\right)^{2}$.

Step 5: calculation of both the Spherical Fuzzy Negative Ideal Solution (SF-NIS), denoted by $X^{-}$, and the Spherical Fuzzy Positive Ideal Solution (SF-PIS), denoted by $X^{+}$, throughout the following expressions, respectively:

$$
\begin{aligned}
& X^{-}:=\left\{\left\langle C_{j}, \min \left\{\operatorname{Score}\left(C_{j}\left(X_{i \omega}\right)\right): \quad i=1, \ldots, m\right\}\right\rangle: j=1, \ldots, n\right\} \\
& =\left\{\left\langle C_{j},\left(\mu_{j}^{-}, v_{j}^{-}, \pi_{j}^{-}\right)\right\rangle: j=1, \ldots, n\right\}, \\
& X^{*}:=\left\{\left\langle C_{j}, \max \left\{\operatorname{Score}\left(C_{j}\left(X_{i \omega}\right)\right) \quad: i=1, \ldots, m\right\}\right\rangle \quad: j=1, \ldots, n\right\} \\
& =\left\{\left\langle C_{j},\left(\mu_{j}^{*}, v_{j}^{*}, \pi_{j}^{*}\right)\right\rangle: j=1, \ldots, n\right\} .
\end{aligned}
$$

Step 6: calculation of the normalized Euclidean distance ([47]) of each alternative $X_{i}$ with respect to the SF-NIS (respectively, the SF-PIS) for all $i=1, \ldots, m$ by means of the next expressions:

$$
\begin{aligned}
& D\left(X_{i}, X^{-}\right)=\sqrt{\frac{1}{2 n} \sum_{j=1}^{n}\left[\left(\mu_{i j}-\mu_{i}^{-}\right)^{2}+\left(v_{i j}-v_{i}^{-}\right)^{2}+\left(\pi_{i j}-\pi_{i}^{-}\right)^{2}\right]}, \\
& D\left(X_{i}, X^{*}\right)=\sqrt{\frac{1}{2 n} \sum_{j=1}^{n}\left[\left(\mu_{i j}-\mu_{i}^{*}\right)^{2}+\left(\nu_{i j}-v_{i}^{*}\right)^{2}+\left(\pi_{i j}-\pi_{i}^{*}\right)^{2}\right]}
\end{aligned}
$$

where $C_{j}\left(X_{i}\right)=\left(\mu_{i j}, v_{i j}, \pi_{i j}\right)$ for all $i=1, \ldots, m$ and all $j=1, \ldots, n$.

Step 7: calculation of the minimum distance with respect to the SF-PIS as well as the maximum distance with respect to the SF-NIS, i.e.,

$$
\begin{gathered}
D_{\min }\left(X_{i}, X^{*}\right)=\min \left\{D\left(X_{i}, X^{*}\right): \quad i=1, \ldots, m\right\}, \\
D_{\max }\left(X_{i}, X^{-}\right)=\max \left\{D\left(X_{i}, X^{-}\right) \quad: i=1, \ldots, m\right\} .
\end{gathered}
$$

Step 8: calculation of the closeness ratio as provided in ([33], equation (37)), thus taking the absolute value of the expression suggested in [48], namely,

$\xi\left(X_{i}\right)=\frac{D\left(X_{i}, X^{*}\right)}{D_{\min }\left(X_{i}, X^{+}\right)}-\frac{D\left(X_{i}, X^{-}\right)}{D_{\max }\left(X_{i}, X^{-}\right)}, \quad$ for all $i=1, \ldots, m$.

Step 9: list the alternatives by increasing the order of their corresponding closeness ratios. In this way, the 
optimal alternative is the one that appears rated in the first position of that ranking.

\section{Assessment of the NEA Deflection Technologies}

3.1. Assumptions of Our Study. We would like to highlight that the primary goal in the current study is to perform a fuzzy MCDM analysis with the aim to assess the following NEA deflection technologies: kinetic impactor (KI), enhanced gravity tractor (EGT), ion-beam deflection (IBD), and laser ablation (LA). Such alternatives will be evaluated with respect to the 8 criteria that have been described in Section 3.3. Furthermore, to deal with that task, the information provided by a group of experts (Section 3.4) will allow us to calculate the aggregated relative importance of a given alternative for each criterion in terms of linguistic labels that are identified with SFNs (Section 4).

The deflection of an asteroid consists of accelerating the object just enough in such a way it crosses Earth's orbit by a minimum distance from the point the NEA would have crossed it providing that it had not been deflected.

The assumptions of our study that were disclosed to the group of experts were as follows. First, we intend to conduct a (nonnuclear) primary deflection greater than or equal to twice Earth' radii (excluding the KI) on a threatening NEA with an estimated diameter lower than or equal to $250 \mathrm{~m}$. Also, the warning time was assumed to range between 5 and 30 years.

We would also like to point out that the assignment of a threatening asteroid to one of the four orbital groups (Apollos, Atens, Atiras, or Amors) has not been specifically considered in the current analysis. Alternatively, and regarding the orbital dynamics of NEAs, they have assumed those assumptions that can be found in [49] and ([50], equation (7)).

The alternatives described in ([19], Section 3.1) and the criteria appeared in ([19], Section 3.2) are also considered throughout this study. Along the next two sections, we summarize them for the sake of completeness.

\subsection{Description of the Alternatives for NEA Deflection}

Alternative $A_{1}$ : the kinetic impactor (KI) consists of placing a spaceship on a trajectory to crash a NEA. This way, both the momentum and the velocity of the targeted asteroid would be modified [49]. It is worth mentioning that it is already possible to impact an asteroid at a high velocity as NASA's Deep Impact mission reported in 2005 [51]. According to the space science community, one of the advantages of the KI deflection technology lies in its immediate effect as well as the high level of momentum that may be delivered to the targeted asteroid. However, there is still a nonnegligible level of uncertainty regarding the amount of momentum that is effectively delivered to the NEA [52].

Alternative $A_{2}$ : the technology under the ion beam deflection (IBD) mainly consists of an ion thruster on board a spacecraft (named the "shepherd") that points a highly collimated high-velocity ion beam at NEA. Simultaneously, a secondary thruster points in the opposite direction to maintain a uniform distance from the asteroid $[49,53]$. In this way, a hovering distance of twice the diameter of the targeted asteroid allows leaving the gravitational force of NEA negligible [54]. Interestingly, the IBD rendezvous spacecraft may be sent to NEA beforehand, which allows decreasing the uncertainty in regard to the orbit of the asteroid. This could be understood as an advantage of the IBD with respect to the KI approach. Moreover, IBD permits an accurate retargeting of the impact point at the asteroid, which becomes especially useful in regard to large asteroids that may be deflected only a few Earth radii (except if a nuclear blast is utilized). Nevertheless, a satisfactory level of autonomy regarding the hovering of the shepherd has not yet been reached. In addition, a greater accuracy concerning the pointing of the beam still lacks [52].

Alternative $A_{3}$ : enhanced gravity tractor (EGT). The gravity tractor (GT) consists of a spaceship that hovers over a targeted NEA being aimed at redirecting its trajectory by taking advantage of the gravitational attraction between the asteroid and the spacecraft. Note that the GT constitutes a trim/observer approach itself [54]. In the case of the enhanced gravity tractor (EGT), the hovering spacecraft increases its mass by removing some rocks or regolith from the targeted NEA. That amount of mass is calculated in such a way that its thrusters at full power and in the general direction of the NEA do not increase the distance between the asteroid and the spaceship. In fact, a uniform separation distance between the spacecraft and the targeted asteroid has to be preserved, so the thrusters slowly impulse the whole system in the opposite direction of the asteroid (to reduce the velocity of the NEA) or in the actual direction of the object (thus improving its velocity) $[49,55]$.

Alternative $A_{4}$ : laser ablation (LA). The energy from the combined effects of a set of phase locked laser amplifiers is continuously impinged on NEA, thus ejecting some material away from its surface and having an effect on the velocity of the targeted asteroid $[49,54,56]$.

3.3. The Selected Attributes. In this study, all the following criteria described will be evaluated by means of scales of importance that are given in terms of SFNs. With this aim, it used the information provided by our group of experts.

Attribute $C_{1}$ : build time. This criterion, $\mathrm{Tb}$, could be understood in the following terms:

$$
\mathrm{Tb}=\text { required warning time }-\mathrm{Tr}-T
$$

where the required warning time is the timeframe from the discovery of the threat to the predicted date of 
collision, $\operatorname{Tr}$ is the rendezvous time, and $T$ is defined for each NEA deflection alternative as follows:

$T= \begin{cases}T_{1}+T_{2}, & \text { if the alternative is either EGT or IBD, } \\ T_{1}, & \text { in the case of LA, } \\ \frac{1}{3} \frac{\Delta X}{\Delta V}, & \text { if the alternative is KI. }\end{cases}$

In equation (12), $T_{1}$ denotes the active deflection time, $T_{2}$ is the coasting time, $\Delta X$ denotes the required deflection distance (in $\mathrm{m}$ ), and $\Delta V$ is the achievable velocity change (in $\mathrm{m} / \mathrm{s}$ ). It should be highlighted that the build time does not include the time each technology needs to achieve the TRL 6 [49]. Observe that the build time is especially important when the warning time is short which, in turn, may be produced by a significant uncertainty concerning the probability of impact of the asteroid with the Earth.

Attribute $C_{2}$ : duration of the active deflection. It is the time needed to achieve a deflection of the targeted asteroid of at least twice Earth' radius (except in the case of the KI).

Attribute $C_{3}$ : asteroid rotation. As it was suggested by our group of experts, it is unlikely to tackle with a fast rotator for objects with estimated diameters ranging $150-240 \mathrm{~m}$.

Attribute $C_{4}$ : asteroid composition. It is worth noting that the efficiency of several NEA deflection approaches may strongly depend on this criterion. For instance, LA may not work appropriately when being applied on metallic surfaces since the heat produced may be conducted away.

Attribute $C_{5}$ : asteroid structure. This is related to the porosity and the internal structure of the object instead of the surface material structure of the asteroid or its friability. It should be pointed out that $\mathrm{KI}$ is sensitive to the internal structure of the object and its porosity, which may affect the momentum transfer. Also, it could influence the ability of EGT to collect material from the NEA surface.

Attribute $C_{6}$ : asteroid shape. A great variety of irregular contours may appear in targeted NEAs.

Attribute $C_{7}$ : level of maturity of a deflection technology or technological readiness level (TRL). This is a standardized scale suggested by NASA to evaluate the current level of development of a technology in regard to a desired maturity level for that approach. In this study, targeted maturity means a redirection technology for asteroids that is ready to be proved in space at the next level, which is equivalent to TRL 6 ([54]).

Attribute $C_{8}$ : mission risk. It takes into account the possibility of a technological failure or an unsuccessful result regarding the asteroid deflection mission. This is quantified separately from the TRL to identify those specific risks that may appear when applying each NEA deflection technique. It is worth mentioning that a scale based on the Goddard risk matrix has been proposed to address the risk assessment ([54, 57-59]).

3.4. Our Group of Experts. A group of 10 researchers whose expertise areas include NEA deflection technologies completed the questionnaires sent by the authors, thus providing some valuable information in regard to the alternatives and criteria involved in our study. Their affiliations were as follows: Langley Research Center and Jet Propulsion Laboratory of the National Aeronautics and Space Administration (three experts), Planetary Defence Office and Galileo Mission of the European Space Agency (two experts), Institute of Space Sciences at the Spanish National Research Council, Institute for Aerospace Studies at the University of Toronto, Department of Physics Applied to Aeronautical Engineering of the Polytechnic University in Madrid, Department of Mathematics and SpaceDyS at the University of Pisa, and Laboratory of Applied Physics at the Johns Hopkins University.

\section{Results and Discussion}

As stated above, the scale of importance appearing in Table 1 ([33]), which identifies a set of linguistic labels with their corresponding SFNs, is considered to assess the criteria and the alternatives involved in the current study. To deal with, the information provided by our advisory board was used. In this way, Table 2 provides the weights of the criteria described in terms of SFNs via the SWAM operator.

The following order of preference regarding our set of criteria holds from the results appeared in Table 2:

$$
C_{1}>C_{2}>C_{8}>C_{4}>C_{7}>C_{5}>C_{3}>C_{6} \text {. }
$$

According to equation (13), $C_{1}$ (build time) appears ranked in the first position being followed by $C_{2}$ (active deflection duration), $C_{8}$ (mission risk), $C_{4}$ (asteroid composition), $C_{7}$ (level of maturity), and $C_{5}$ (asteroid structure). Then, $C_{3}$ (asteroid rotation) and $C_{6}$ (asteroid shape) are found with a same level of importance. In this regard, a good reference to identify a set of linguistic labels with their corresponding SFNs can be found in [33].

When applying the triangular fuzzy set (TFS, hereafter) version of the analytic hierarchy process (AHP) approach ([19]), the next order of preference was found for our set of criteria by means of the valuable information provided by the group of experts:

$$
C_{1}>C_{2}>C_{7}>C_{4}>C_{5}>C_{8}>C_{6}>C_{3} .
$$

Hence, from both equations (13) and (14), it holds that the criteria $C_{7}$ (level of maturity) and $C_{4}$ (asteroid composition) interchange their relative level of importance from the SWAM operator to the TFS version of AHP. Specifically, it holds that $C_{4}$ appears as a more important criterion than $\mathrm{C}_{7}$ when applying SFS TOPSIS. It is also worth pointing out that the attribute $C_{8}$ (mission risk) has been assigned a 
TABLE 2: Weights of the criteria in terms of SFNs.

\begin{tabular}{lccr}
\hline Spherical fuzzy numbers & & Weights \\
Criteria & $\mu$ & $\nu$ & 0.2 \\
\hline$C_{1}$ (build time) & 0.8 & 0.5 & 0.2 \\
$C_{2}$ (active deflection duration) & 0.6 & 0.6 & 0.4 \\
$C_{3}$ (asteroid rotation) & 0.4 & 0.4 & 0.4 \\
$C_{4}$ (asteroid composition) & 0.6 & 0.5 & 0.4 \\
$C_{5}$ (asteroid structure) & 0.5 & 0.6 & 0.4 \\
$C_{6}$ (asteroid shape) & 0.4 & 0.4 & 0.4 \\
$C_{7}$ (level of maturity) & 0.6 & 0.5 & 0.3 \\
$C_{8}$ (mission risk) & 0.6 & 0.3 \\
\hline
\end{tabular}

greater level of importance when applying the SWAM operator than TFS AHP.

The next step was to generate a new decision matrix (Table 3) that contains the assessment of the alternatives for such criteria from the judgments provided by the experts via linguistic labels defined in terms of SFNs (Table 1).

From that decision matrix and taking into account the weights of the criteria (obtained by the SWAM operator), the SFS TOPSIS methodology was applied to rank the alternatives of our case of study. In this way, Table 4 displays a comparison between the rankings provided by the SFS TOPSIS approach vs. the one obtained by means of TFS TOPSIS methodology.

The SFS TOPSIS-based ranking in Table 4 shows that the alternatives LA and EGT do interchange their positions with respect to their TFS TOPSIS rankings. This could be due to the greater SFS TOPSIS relative importance that has been assigned to the criterion $C_{4}$ (asteroid composition) to the detriment of $C_{7}$ (level of maturity). In fact, a greater valuation for that criterion (Table 3) places LA with respect to $C_{4}$, thus being followed by KI, IBD, and EGT.

Similarly, since mission risk (criterion $C_{8}$ ) for both alternatives LA and EGT is greater than the one for both KI and IBD, and it was the 3rd most important criterion according to SWAM operator (equation (13)), both LA and EGT become closer from KI and IBD in the SFS TOPSIS ranking.

\section{Sensitivity Analyses}

Two sensitivity analyses have been carried out in this section with the aim to validate the robustness of the results provided in Section 4. In fact, the first one consists of carrying out the SFS TOPSIS calculations by means of the SWGM operator and taking into account the weights of the criteria as provided by the judgments from the group of experts (Section 5.1), whereas the second sensitivity analysis repeats the SFS TOPSIS calculations by both operators, SWAM and SWGM, but assuming that the weights of all the criteria are the same. Two interesting facts follow from the results provided by each sensitivity analysis.

5.1. On the Effect of the SWGM Operator. Recall that arithmetic mean is used to aggregate the valuations provided by the experts to generate the decision matrix of the TOPSIS approach (e.g., [19]). However, when ranking the alternatives through the SFS TOPSIS procedure, we can use either the SWAM operator or the SWGM operator, which constitutes an advantage of SFSs over TFSs to deal with fuzzy series. In fact, applying geometric mean to generate the aggregated matrix of decision (by scales of importance through TFS) could provoke that the TOPSIS algorithm may not be executed.

In this section, we compare the ranking of alternatives provided by the SFS TOPSIS approach and the SWAM operator (Section 4) vs. the one provided by the SFS TOPSIS approach when the SWAM operator is applied. In both cases, the weights of the criteria were calculated according to the information provided by our group of experts. We found that both rankings of alternatives were found to be the same (Table 5), which suggests that the choice of the SWGM (respectively, the SWAM) operator does not influence the ranking positions of the alternatives. Only slight deviations were found in regard to the absolute values of the differences between the closeness ratios of pairs of consecutive alternatives. As such, the choice of one of such operators to the detriment of the other would mainly depend on the computational cost required to carry out the corresponding calculations. However, in this case, the computational cost is similar for both operators.

5.2. On the Dependence of the SFS TOPSIS Approach on the Judgments from the Group of Experts. Next, we highlight the influence of the information provided by the group of experts over our SFS TOPSIS rankings of alternatives. With this aim, a pair of SFS TOPSIS rankings was obtained (one per each operator, SWAM and SWGM) by assuming that the weights of all the criteria are the same. First, as shown in Table 6, it holds that the positions of the four alternatives involved in the present study were found to be the same in both SFS TOPSIS-based rankings. However, such SFS TOPSIS-based rankings differ from the one provided in [19], where TFS TOPSIS was used to assess these four NEA deflection technologies. In fact, the use of one of such operators (SWAM or SWGM) may lead to some changes in regard to the rankings of alternatives as provided by the SFS TOPSIS approach with respect to the rankings of alternatives provided by the TFS TOPSIS procedure.

Specifically, observe that KI keeps the first position in all such SFS TOPSIS-based rankings. On the other hand, LA is ranked in 3rd position in both SFS TOPSIS rankings when the weights of the criteria are calculated from the group of 
TABLE 3: Assessment of the alternatives for criteria $C_{1}-C_{8}$ in terms of SFNs as provided by the SFS TOPSIS approach via the SWAM operator.

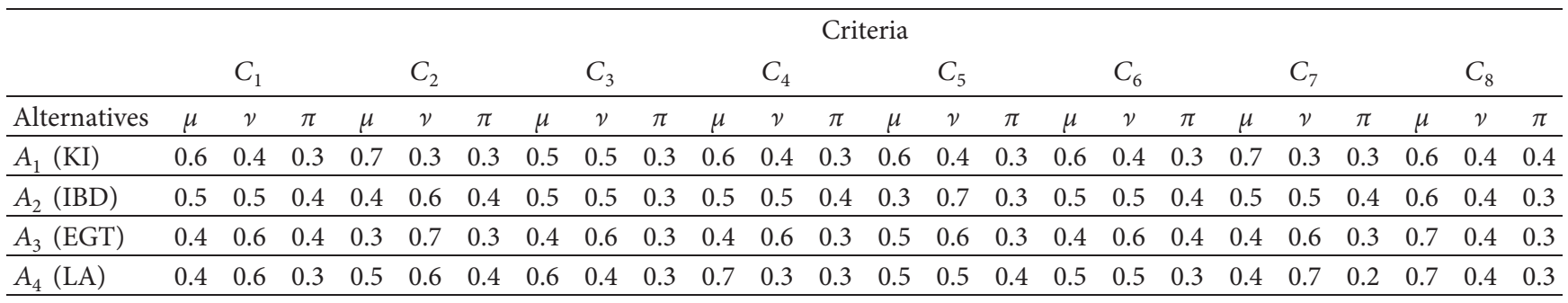

TABLE 4: Comparison of rankings of alternatives between the SFS TOPSIS (SWAM operator) approach to TFS TOPSIS procedure ([19]). The weights of the criteria were obtained from the information provided by the group of experts.

\begin{tabular}{lcccc}
\hline \multirow{2}{*}{ Alternative } & \multicolumn{2}{c}{ SFS TOPSIS (SWAM) ranking } & \multicolumn{2}{c}{ TFS TOPSIS ranking } \\
& Closeness ratio & Ranking & $R_{i}$ & 3.07 \\
\hline$A_{1}$ (KI) & 0.00 & 1 & 1.85 & 1 \\
$A_{2}$ (IBD) & 5.39 & 2 & 1.16 & 2 \\
$A_{3}$ (EGT) & 7.82 & 4 & 0.74 & 3 \\
$A_{4}$ (LA) & 5.99 & 3 & 4 \\
\hline
\end{tabular}

TABLE 5: SFS TOPSIS (SWGM) ranking of alternatives as described in the first analysis of sensitivity. The weights of the criteria were chosen to be those calculated from the information provided by the group of experts. The "Diff." column contains the differences between the closeness ratios (in absolute value) from pairs of alternatives.

SFS TOPSIS (SWGM) ranking of alternatives (criteria weights from experts)

\begin{tabular}{lccc} 
Alternative & Closeness ratio & Rank & Diff. \\
\hline$A_{1}(\mathrm{KI})$ & 0.00 & 1 & - \\
$A_{2}(\mathrm{IBD})$ & 3.24 & 2 & 3.24 \\
$A_{3}$ (EGT) & 4.81 & 4 & 1.57 \\
$A_{4}(\mathrm{LA})$ & 3.86 & 3 & 0.95 \\
\hline
\end{tabular}

TABLE 6: SFS-based rankings of alternatives for both operators, SWAM and SWGM, under the assumption that the all the criteria are equally weighted (Section 5.2) vs. TFS-based ranking of alternatives for equally weighted criteria ([19]).

\begin{tabular}{lccc}
\hline \multirow{2}{*}{ Alternative } & \multicolumn{2}{c}{ SFS rankings (equally weighted criteria) } & \multicolumn{2}{c}{ TFS ranking (equally weighted criteria) } \\
& SWAM rank & SWGM rank & 1 \\
\hline$A_{1}(\mathrm{KI})$ & 1 & 1 & 2 \\
$A_{2}$ (IBD) & 3 & 3 & 3 \\
$A_{3}$ (EGT) & 4 & 4 & 4 \\
$A_{4}$ (LA) & 2 & 2 & 4 \\
\hline
\end{tabular}

experts, though it appears ranked in 4th position in the TFS TOPSIS ranking for equally weighted criteria. However, it occupies 2nd position in both SFS TOPSIS rankings for equally weighted criteria. The next ranked alternatives are IBD and EGT (notice that such a consecutive order for such alternatives coincides with the one that appears in the TFS TOPSIS-based rankings).

This analysis of sensitivity highlights that, unlike the TFS TOPSIS procedure, the weights of the criteria should be assigned carefully when applying a SFS TOPSIS approach since variations regarding the weights of the criteria may induce changes of positions among the ranked alternatives.

\section{Conclusions}

In this section, we summarize the main conclusions to be highlighted from the study carried out.

First of all, it is worth pointing out that the KI alternative is consolidated as the best choice for active NEA deflection purposes. In fact, the results thrown by the SFS TOPSIS methodology coincide with all those presented in [19] when it was applied to the TFS TOPSIS approach with the same purpose.

However, this study highlights the fact that a SFS TOPSISbased ranking of alternatives may vary widely when a sensitivity 
analysis is carried out. Specifically, we showed that the ranking of alternatives as provided by the SFS TOPSIS approach when taking into account the information from the group of experts becomes quite different from the SFS TOPSIS ranking of alternatives we obtained provided that all the weights of the criteria are assumed to be the same. In other words, this study reveals a nonnegligible dependence of the SFS TOPSIS results from the judgments that could be provided by the group of experts with the aim of ranking a set of alternatives.

On the other hand, we would like to mention that the use of either the SWAM operator or the SWGM operator is indifferent when carrying out SFS TOPSIS calculations. In fact, only slight differences between the absolute value of the closeness ratios from pairs of consecutive alternatives were found with a similar computational cost. This fact could be understood as an advantage of SFS TOPSIS approach to the detriment of TFS TOPSIS. In fact, the latter only uses arithmetic mean in contexts where it is necessary to utilize the lowest level of the standard TFS scale of importance.

Note that a consistency analysis regarding the judgments provided by the group of experts cannot be carried out through the SWAM (respectively, the SWGM) operator. Notwithstanding, recently, it has been contributed in [60] a SFS version of the AHP methodology, which encourages us to calculate the weights of the criteria throughout that novel approach as a future research task. Also, we would like to carry out additional comparative analyses between both SFS and TFS approaches, especially to address other decision problems of astronomical interest, with the aim to investigate whether our conclusions also recur in other cases of the study.

\section{Data Availability}

The data used to support the findings of this study (i.e., the information provided by the group of experts that are filled in the questionnaires based on the methodologies applied in this work) are available from the corresponding author upon request.

\section{Disclosure}

The survey' results are based on the expert opinions of the participating individuals and do not necessarily reflect the official positions of their parent institutions.

\section{Conflicts of Interest}

The authors declare that they have no conflicts of interest.

\section{Acknowledgments}

This research has been partially funded by the research project (PGC2018-097374-B-I00), funded by FEDER/Ministerio de Ciencia e Innovación - Agencia Estatal de Investigación. M.F.M. appreciates the partial support of Ministerio de Ciencia, Innovación y Universidades, grant number PGC2018-097198-B-I00, and Fundación Séneca of Región de Murcia, grant number 20783/PI/18. J.M.S.L. acknowledges the support of Grants TIN2017-86647-P and 19882-GERM-15 from the Spanish Ministry of Economy and Competitiveness (MINECO) and Fundación Séneca (Región de Murcia), respectively. This work could have not been carried out without the generous collaboration of experts from the following institutions: Langley Research Center and Jet Propulsion Laboratory of the National Aeronautics and Space Administration (NASA), Planetary Defence Office and Galileo Mission of the European Space Agency (ESA), Laboratory of Applied Physics at the Johns Hopkins University, Institute for Aerospace Studies at the University of Toronto, Institute of Space Sciences at the Spanish National Research Council, Department of Physics Applied to Aeronautical Engineering of the Polytechnic University in Madrid, and the Department of Mathematics and SpaceDyS at the University of Pisa. The survey results are based on the expert opinions of the participating individuals and do not necessarily reflect the official positions of their parent institutions. The authors would also like to express their gratitude to the editor and anonymous reviewers whose suggestions, comments, and remarks have allowed them to enhance the quality of this paper.

\section{References}

[1] D. E. Brownlee, "The origin and properties of dust impacting the Earth," in Accretion of Extraterrestrial Matter Throughout Earth's History, B. Peucker-Ehrenbrink and B. Schmitz, Eds., pp. 1-12, Kluwer Academic/Plenum, New York, NY, USA, 2001.

[2] N. Anthony and M. R. Emami, "Asteroid engineering: the state-of-the-art of near-earth asteroids science and technology," Progress in Aerospace Sciences, vol. 100, pp. 1-17, 2018.

[3] R. A. F. Grieve, "Logan medallist 4. Large-scale impact and earth history," Geoscience Canada, vol. 44, no. 1, pp. 1-26, 2017.

[4] M. B. E. Boslough and D. A. Crawford, "Shoemaker-levy 9 and plume-forming collisions on earth," Annals of the New York Academy of Sciences, vol. 822, no. 1, pp. 236-282, 1997.

[5] S. W. Paek, O. de Weck, J. Hoffman, R. Binzel, and D. Miller, "Optimization and decision-making framework for multistaged asteroid deflection campaigns under epistemic uncertainties," Acta Astronautica, vol. 167, pp. 23-41, 2020.

[6] O. P. Popova, P. Jenniskens, V. Kartashova et al., "Chelyabinsk airburst, damage assessment, meteorite recovery, and characterization," Science, vol. 342, no. 6162, pp. 1069-1073, 2013.

[7] M. B. E. Boslough, "Tsunami from plume-forming collisional airbursts," IAA Planetary Defense Conference, Sandia National Laboratories (SNL-NM), Albuquerque, NM, USA, 2013.

[8] M. I. Gritsevich, V. P. Stulov, and L. I. Turchak, "Consequences of collisions of natural cosmic bodies with the Earth's atmosphere and surface," Cosmic Research, vol. 50, no. 1, pp. 56-64, 2012.

[9] H. Hammel, R. Beebe, A. Ingersoll et al., "HST imaging of atmospheric phenomena created by the impact of comet shoemaker-levy 9," Science, vol. 267, no. 5202, pp. 1288-1296, 1995.

[10] J. G. Hills and M. P. Goda, "The fragmentation of small asteroids in the atmosphere," The Astronomical Journal, vol. 105, no. 3, pp. 1114-1144, 1993. 
[11] S. Glasstone and P. J. Dolan, The Effects of Nuclear Weapons, Department of Defense, Department of Energy, Washington, DC, USA, 1977.

[12] E. A. Silber, M. Boslough, W. K. Hocking, M. Gritsevich, and R. W. Whitaker, "Physics of meteor generated shock waves in the Earth's atmosphere-a review," Advances in Space Research, vol. 62, no. 3, pp. 489-532, 2018.

[13] NEO Basics, Center for Near Earth Object Studies (CNEOS)Jet Propulsion Laboratory, California Institute of Technology and NASA, Pasadena, CA, USA, 2020, https://cneos.jpl.nasa.gov/ about/neo_groups.html.

[14] N. Thiry and M. Vasile, "Statistical multi-criteria evaluation of non-nuclear asteroid deflection methods," Acta Astronautica, vol. 140, pp. 293-307, 2017.

[15] C. R. Chapman, "The hazard of near-Earth asteroid impacts on earth," Earth and Planetary Science Letters, vol. 222, no. 1, pp. 1-15, 2004.

[16] Asteroid Redirect Mission, NASA, 2020, https://www.nasa. gov/content/what-_is-_nasa-_s-_asteroid-_redirect-_ mission.

[17] New Space Policy Directive Calls for Human Expansion across Solar System, 2020, https://www.nasa.gov/press-_ release/new-_space-_policy-_directive-_calls-_for-_human-_ expansion-_across-_solar-_system.

[18] Double Asteroid Redirection Test (DART) Mission, NASA, 2020, https://www.nasa.gov/planetarydefense/dart.

[19] J. M. Sánchez-Lozano, M. Fernández-Martínez, A. A. Saucedo-Fernández, and J. M. Trigo-Rodriguez, "Evaluation of NEA deflection techniques. A fuzzy multicriteria decision making analysis for planetary defense," Acta Astronautica, vol. 176, pp. 383-397, 2020.

[20] J. M. Sánchez-Lozano, M. Fernández-Martínez, and M. T. Lamata, "Near-Earth asteroid impact dates: a reference ideal method (RIM) approach," Engineering Applications of Artificial Intelligence, vol. 81, pp. 157-168, 2019.

[21] J. M. Sánchez-Lozano and M. Fernández-Martínez, "NearEarth object hazardous impact: a Multi-Criteria Decision Making approach," Scientific Reports, vol. 6, Article ID 37055, 2016.

[22] L. A. Zadeh, "Fuzzy sets," Information and Control, vol. 8, no. 3, pp. 338-353, 1965.

[23] A. Mardani, A. Jusoh, and E. K. Zavadskas, "Fuzzy multiple criteria decision-making techniques and applications-two decades review from 1994 to 2014," Expert Systems with Applications, vol. 42, no. 8, pp. 4126-4148, 2015.

[24] K. T. Atanassov, "Intuitionistic fuzzy sets," Fuzzy Sets and Systems, vol. 20, no. 1, pp. 87-96, 1986.

[25] R. R. Yager, "Pythagorean fuzzy subsets," in Proceedings of the 2013 Joint IFSA World Congress and NAFIPS Annual Meeting (IFSA/NAFIPS), pp. 57-61, Edmonton, AB, Canada, June 2013.

[26] R. R. Yager, "Pythagorean membership grades in multicriteria decision making," IEEE Transactions on Fuzzy Systems, vol. 22, no. 4, pp. 958-965, 2014.

[27] R. R. Yager, "On the theory of bags," International Journal of General Systems, vol. 13, no. 1, pp. 23-37, 1986.

[28] K. Atanassov and G. Gargov, "Interval valued intuitionistic fuzzy sets," Fuzzy Sets and Systems, vol. 31, no. 3, pp. 343-349, 1989.

[29] F. Smarandache, A Unifying Field in Logics: Neutrosophic Logic. Neutrosophy, Neutrosophic Set, Neutrosophic Probability and Statistics, InfoLearnQuest, Philadelphia, PA, USA, 6th edition, 2007.
[30] S. Zeng, S. Asharf, M. Arif, and S. Abdullah, "Application of exponential jensen picture fuzzy divergence measure in multicriteria group decision making," Mathematics, vol. 7, no. 2, p. 191, 2019.

[31] B. C. Cuong, "Picture fuzzy sets," Journal of Computer Science and Cybernetics, vol. 30, no. 4, pp. 409-420, 2014.

[32] S. Ashraf and S. Abdullah, "Spherical aggregation operators and their application in multiattribute group decision-making," International Journal of Intelligent Systems, vol. 34, no. 3, pp. 493-523, 2019.

[33] F. K. Gündoğdu and C. Kahraman, "Spherical fuzzy sets and spherical fuzzy TOPSIS method," Journal of Intelligent \& Fuzzy Systems, vol. 36, no. 1, pp. 337-352, 2019.

[34] Y. Jin, S. Ashraf, and S. Abdullah, "Spherical fuzzy logarithmic aggregation operators based on entropy and their application in decision support systems," Entropy, vol. 21, no. 7, p. 628, 2019.

[35] S. Ashraf, S. Abdullah, M. Aslam, M. Qiyas, and M. A. Kutbi, "Spherical fuzzy sets and its representation of spherical fuzzy t-norms and t-conorms," Journal of Intelligent \& Fuzzy Systems, vol. 36, no. 6, pp. 6089-6102, 2019.

[36] H. Jin, S. Ashraf, S. Abdullah, M. Qiyas, M. Bano, and S. Zeng, "Linguistic spherical fuzzy aggregation operators and their applications in multi-attribute decision making problems," Mathematics, vol. 7, no. 5, p. 413, 2019.

[37] S. Ashraf, S. Abdullah, and L. Abdullah, "Child development influence environmental factors determined using spherical fuzzy distance measures," Mathematics, vol. 7, no. 8, p. 661, 2019.

[38] M. Rafiq, S. Ashraf, S. Abdullah, T. Mahmood, and S. Muhammad, "The cosine similarity measures of spherical fuzzy sets and their applications in decision making," Journal of Intelligent \& Fuzzy Systems, vol. 36, no. 6, pp. 6059-6073, 2019.

[39] J. M. Sánchez-Lozano and O. Naranjo Rodríguez, “Application of Fuzzy Reference Ideal Method (FRIM) to the military advanced training aircraft selection," Applied Soft Computing Journal, vol. 88, Article ID 106061, 2020.

[40] L. A. Zadeh, "The concept of a linguistic variable and its application to approximate reasoning-I," Information Sciences, vol. 8, no. 3, pp. 199-249, 1975.

[41] K. T. Atanassov, "Geometrical interpretation of the elements of the intuitionistic fuzzy objects, mathematical foundations of artificial intelligence seminar, Sofia," International Journal Bioautomation, vol. 20, no. S1, pp. S27-S42, 1989.

[42] F. K. Gündoğdu and C. Kahraman, "A novel fuzzy TOPSIS method using emerging interval-valued spherical fuzzy sets," Engineering Applications of Artificial Intelligence, vol. 85, pp. 307-323, 2019.

[43] Z. Gong, X. Xu, Y. Yang, Y. Zhou, and H. Zhang, "The spherical distance for intuitionistic fuzzy sets and its application in decision analysis," Technological and Economic Development of Economy, vol. 22, no. 3, pp. 393-415, 2016.

[44] Y. Yang and F. Chiclana, "Intuitionistic fuzzy sets: spherical representation and distances," International Journal of Intelligent Systems, vol. 24, no. 4, pp. 399-420, 2009.

[45] B. Oztaysi, C. Kahraman, S. C. Onar, and I. Otay, "Indoor location tracking technology evaluation by using spherial fuzzy TOPSIS method," Developments of Artificial Intelligence Technologies in Computation and Robotics, vol. 24, pp. 173$181,2020$.

[46] F. K. Gündoğdu and C. Kahraman, "Optimal site selection of electric vehicle charging station by using spherical fuzzy TOPSIS method," in Decision Making with Spherical Fuzzy 
Sets. Studies in Fuzziness and Soft Computing, C. Kahraman and F. Kutlu Gündoğdu, Eds., vol. 392, Cham, Switzerland, Springer, 2021.

[47] E. Szmidt and J. Kacprzyk, "Distances between intuitionistic fuzzy sets," Fuzzy Sets and Systems, vol. 114, no. 3, pp. 505-518, 2000.

[48] X. Zhang and Z. Xu, "Extension of TOPSIS to multiple criteria decision making with pythagorean fuzzy sets," International Journal of Intelligent Systems, vol. 29, no. 12, pp. 1061-1078, 2014.

[49] C. Weisbin, W. Lincoln, B. Wilcox, J. Brophy, P. Chodas, and B. Muirhead, "Comparative analysis of asteroid-deflection approaches," in Proceedings of the 2015 IEEE Aerospace Conference, pp. 1-16, Big Sky, MT, USA, March 2015.

[50] T. J. Ahrens and A. W. Harris, "Deflection and fragmentation of near-Earth asteroids," Nature, vol. 360, no. 6403, pp. 429-433, 1992.

[51] NASA, Deep Impact Website, 2020, https://www.jpl.nasa.gov/ missions/deep-_impact/.

[52] C. Bombardelli, E. J. Calero, and J. L. Gonzalo, "Deflection of fictitious asteroid 2017 PDC: ion beam vs. kinetic impactor," Acta Astronautica, vol. 156, pp. 301-307, 2019.

[53] C. Bombardelli and J. Peláez, "Ion beam shepherd for asteroid deflection," Journal of Guidance, Control, and Dynamics, vol. 34, no. 4, pp. 1270-1272, 2011.

[54] M. C. F. Bazzocchi and M. R. Emami, "Comparative analysis of redirection methods for asteroid resource exploitation," Acta Astronautica, vol. 120, pp. 1-19, 2016.

[55] C. R. McInnes, "Near earth object orbit modification using gravitational coupling," Journal of Guidance, Control, and Dynamics, vol. 30, no. 3, pp. 870-873, 2007.

[56] M. Vasile and C. A. Maddock, "Design of a formation of solar pumped lasers for asteroid deflection," Advances in Space Research, vol. 50, no. 7, pp. 891-905, 2012.

[57] J. R. Wertz, D. F. Everett, and J. J. Puschell, Space Mission Engineering: The New SMAD, Microcosm Press, Hawthorne, CA, USA, 2011.

[58] J. C. Mankins, "Technology readiness and risk assessments: a new approach," Acta Astronautica, vol. 65, no. 9-10, pp. 1208-1215, 2009.

[59] NASA, Risk Management Reporting-GSFC-STD-0002, Tech. Report, Goddard Space Flight Center, Greenbelt, MD, USA, 2009.

[60] B. Oztaysi, S. C. Onar, and C. Kahraman, “A dynamic pricing model for location based systems by using spherical fuzzy AHP scoring," Journal of Intelligent \& Fuzzy Systems, vol. 39, no. 5, pp. 6293-6302, 2020. 